\section{General Paediatrics}

\section{G99 HOW DO PAEDIATRICIANS COMMUNICATE WITH CHILDREN?}

E. Wassmer, N. Abdel Aal, M. Atkinson, E. Gupta, G. Minnaar, S. Yuen, G. Rylance. General Paediatrics Department, Children's Hospital, Birmingham, UK

The outpatient clinic visit is the major focus of the hospital medical process for most paediatricians, children and parents. The importance of children as active participants has been recognised. This study aimed to describe and assess the components of doctor-parent-child communication in the outpatient setting.

Fifty-one medical paediatric clinic consultations were audiorecorded and communication was analysed according to quantitive and qualitive methods. Questionnaires assessed parental and children's perception.

Doctors contributed most to the conversation (55.9\%), children spoke only $4.2 \%$ of the total consultation time.

Content: Doctors' communication consisted of $84 \%$ instrumental behaviour (58\% informative, $20 \%$ assertive i.e. asking questions and $6 \%$ directive i.e. giving instructions), 13\% affective (expressing concerns and worries) and 3\% social (small talk). Parental communication was $83 \%$ informative, $13 \%$ assertive and $4 \%$ social. Children's communication was less assertive (3\%) and more social $(19 \%)$.

Control: Doctors dominated in turn taking ( $52 \%)$. Children took $9 \%$ of all turns. The doctors asked most of the questions ( $85 \%)$. Children asked $2 \%$ of the questions.

Language: Doctors used little medical jargon (17 technical terms in 51 consultations), $50 \%$ were explained.

Children and parental perception: $95 \%$ children and $97 \%$ parents were satisfied with their doctor's communication skills.

Communication is mainly instrumental. More affective communication is required to meet emotional needs. Doctors tend to dominate and direct the interview. Children's contribution is small. The participation of children needs to be encouraged for communication to be more patient-centred.

\section{G100 HOW LIKELY IS MENINGOCOCCAL DISEASE IN A CHILD WITH A NON-BLANCHING RASH: A PROSPECTIVE COHORT STUDY}

L.C. Wells, J.C. Smith, V.C. Weston, J. Collier, N. Rutter. Queens Medical Centre, Nottingham, UK

Objectives: to examine a number of simple clinical features and investigations in children with a non-blanching rash to see which might predict the likelihood of meningococcal infection.

Design: A prospective observational cohort study

Subjects: 233 infants and children up to 15 years of age presenting with a non-blanching rash over a period of twelve months

Setting: The children's accident and emergency department of a large teaching hospital

Method: Clinical features and laboratory investigations were recorded at presentation. The ability of each to predict meningococcal infection was examined.

Statistics: The specificity, sensitivity, positive and negative predictive values of each feature were calculated.

Results: $11 \%$ had proven meningococcal infection. Children with meningococcal infection were more likely to be ill $(19 / 24)$, pyrexial $\left(>37.5^{\circ} \mathrm{C}\right)(19 / 24)$, to have purpura $(20 / 24)$ and a capillary refill time of more than two seconds $(20 / 24)$ than non-meningococcal children. No child with a rash confined to the distribution of the superior vena cava $(74 / 218)$ had meningococcal infection. Investigations were less helpful, although children with meningococcal infection were more likely to have an abnormal neutrophil count (15/24) and a prolonged INR (14/24). Many children with meningococcal infection (15/24) had a CRP of 100 or more. No child with a CRP of less than $6 \mathrm{mg} / 1$ (90/183) had meningococcal infection.

Conclusions and recommendations: Most children with meningococcal infection are ill, have a purpuric rash, a fever and delayed capillary refill. They should be admitted to hospital and treated without delay. Children with a non-blanching rash confined to the distribution of the superior vena cava are very unlikely to have meningococcal infection and their parents can be safely reassured. Measurement of CRP may be helpful - no child with a normal value had meningococcal infection.

\section{G101 A MATCHED CONTROLLED TRIAL OF MODIFIED DRY-BED TRAINING (M-DBT) AND URINE ALARM CONDITIONING (UAC)}

D. Tappin ${ }^{1}$, S. Nawaz 2 , P.V. Griffiths ${ }^{2} .{ }^{1}$ Paediatric Epidemiology \& Community Health Unit, Department of Child Health, Glasgow University; ${ }^{2}$ Department of Psychology, Stirling University, UK

Aims: To compare acceptability and efficacy of M-DBT and UAC to treat monosymptomatic nocturnal enuresis in deprived children aged 7-12 years attending community based clinics in Glasgow.

Method: M-DBT uses a mat in the child's bed attached by long cable to an alarm near the parent. A single Intensive Training Night, with hourly wakening, is followed by Post-Training Supervision, when cleanliness training and positive practice are contingent upon a wet bed. UAC has an alarm by the child's bed. Treatment manuals and videos were prepared for both M-DBT and UAC. Between November 1999 and July 2000, 36 new families were consecutively assigned to three groups M-DBT, UAC and waiting list controls, with matching for age and sex. Initial interview was followed by two review appointments. Fortnightly telephone support continued for 16 weeks or until initial success (14 consecutive dry nights). Six month follow-up assessed relapse.

Results: 8 of 12 using M-DBT achieved initial success compared with 3 of 12 with UAC. One control had spontaneous remission. One-way analysis of variance $(\mathrm{p}<0.001)$ showed a highly significant difference between any treatment and control. M-DBT was acceptable to deprived families.

Conclusion: M-DBT should be a first line treatment for nocturnal enuresis. To establish effectiveness, school nurses need to apply M-DBT with process and outcome evaluation.

\section{G102 PULSE OXIMETRY AS A SCREENING TEST IN NEONATES}

M.G. Gnanalingham, B.M. Mehta, M. Siverajan, N.J. Wild, C.D. Bedford Neonatal Department, Warrington General Hospital, Lovely Lane, Warrington WAS $1 Q G, U K$

Aims: To determine the effectiveness of pulse oximetry as a screening test in the early detection of serious illness in neonates, in particular cyanotic congenital heart disease (CCHD) and early onset sepsis.

Methods: From 1st September 1999 to 31st August 2000, midwives in our Maternity Unit determined the oxygen saturations of live born babies who did not require immediate transfer to the Neonatal Unit. The oxygen saturations were recorded between 0.5 and 6 hours of age on one foot using either a Radiometer ${ }^{\circledR}$ or Novametrix ${ }^{\circledR}$ pulse oximeter.

Results: During the 12-month study period, there were 3218 deliveries that met the inclusion criteria. Saturations were recorded in $1421(44.2 \%)$. An inability to obtain a satisfactory saturation trace and patient transfer prior to saturation readings were common reasons cited for no initial saturation readings. The mean saturation was $96.6 \pm 3.8$ (SD) \%, mean time of recording was $1.7 \pm 0.7$ hours, mean gestational age was $39.4 \pm 1.8$ weeks and mean birthweight was $3416 \pm 532$ grams. The initial saturation was $\leqslant 92 \%$ in $81(6 \%)$ cases, $\leqslant 91 \%$ in $46(3 \%)$ cases and $\leqslant 90 \%$ in $31(2 \%)$ cases. Of the latter 31 cases, $18(58 \%)$ were clinically normal on review and had subsequent satisfactory saturations. Of the remaining 13 cases, 1 had a disconnected right pulmonary artery with no clinical evidence of CCHD, 8 had transient tachypnoea of the newborn; 2 had Group B Streptococcal infection and 2 had symptomatic hypoglycaemia. There were no missed cases of serious illness that presented in the 7 days following birth. One case of transposition of great arteries and one case of persistent ductus arteriosus, ventricular septal defect and reversible pulmonary hypertension were admitted to the Neonatal Unit from the postnatal ward. Neither case had their initial saturation recorded.

Conclusions: With an oxygen saturation cut-off of $\leqslant 90 \%$ in air, we identified 31 babies of whom $3(10 \%)$ cases had serious illness, 
who otherwise appeared healthy. The pulse oximeter can be an effective screening test for the early detection of serious illness, including CCHD and early onset sepsis.

\section{G103 BLOODSTAINED CEREBROSPINAL FLUID-IS THERE A REASON TO WORRY?}

I. Chandrasekar, F.A.I. Riordan, M. Honeyman. Department of Child Health, Birmingham Heartlands Hospital, Birmingham, UK

Introduction: Bloodstained cerebrospinal fluid (BSCSF) usually occurs due to local trauma during lumbar puncture. It may also occur in children with non-accidental head injury (NAHI). There is little data on the frequency of BSCSF in children or the proportion due to NAHI.

Aims: 1 To determine the frequency of BSCSF in children undergoing lumbar puncture. 2.To determine the proportion of children with BSCSF who have NAHI. 3. To evaluate features which suggest $\mathrm{NAHI}$ in these children.

Method: All cerebrospinal fluid results from children admitted to the children's wards, between April 1998 and March 2000, were obtained from the microbiology database. BSCSF was defined as $>250 \mathrm{RBC} / \mu \mathrm{l}$. Medical records of children less than 2 years of age with BSCSF were reviewed.

Results: CSF was obtained from 196 children over the 2-year period, $61(31 \%)$ of these were bloodstained. BSCSF was more common in children under 1 year of age compared with older children (41/92 vs $20 / 104 ; \mathrm{P}=0.0001)$. 56 of $127(44 \%)$ children under 2 years of age had BSCSF. The median age in this group was 5 weeks. Of these $56,3(5 \%)$ had NAHI. In 2 infants a CT scan detected this during admission. The third infant did not have a CT scan and re-presented a week later with extensive subdural haemorrhages and died. All three infants had either signs suggesting child abuse (bruising/retinal haemorrhages) or a haemoglobin $<9 \mathrm{grm} / \mathrm{dl}$ and a platelet count $>500 \times 10^{\circ}$. This combination of anaemia and raised platelets was present in only one other infant with BSCSF, who had a normal CT scan. Xanthochromia was reported in samples from 6 children, none had NAHI.

Conclusion: Cerebrospinal fluid is blood stained in $45 \%$ of infants during lumbar puncture. One in 20 children under two years of age with BSCSF may have NAHI. Infants with BSCSF should be carefully examined for signs of child abuse. Anaemia and raised platelets may indicate NAHI in an infant with BSCSF and no other signs of abuse.

\section{G104 THE CONTINUING PROBLEM OF VITAMIN D DEFICIENCY IN PREGNANT ASIAN WOMEN AND THEIR OFFSPRING: AN INTERFACE AUDIT AS A PRELUDE TO ACTION}

S.J. Iqbal ${ }^{1}$, C. Walker ${ }^{2}$, P.G.F. Swift ${ }^{3}$. Dept of Chemical Pathology ${ }^{1}$, Clinical Effectiveness ${ }^{2}$, Childrens Hospital ${ }^{3}$, Leicester Royal Infirmary, UHL NHS Trust, $U K$

Vitamin D deficiency is well documented in UK Asians especially in pregnancy and infancy. The Leicestershire population $(865,000)$ includes $8.9 \%$ South Asians. In the past 3 years, 17 paired cases of maternal vitamin D deficiency and neonatal hypocalcaemic fits have been seen and infantile rickets remains endemic. Supplementation of pregnant women has been recommended but there is no formal policy.

Method: Questionnaire sent to Family Doctors (F 512), Hospital Doctors involved in antenatal care (H 32) and Midwives (M 212). Replies are expressed as percentages of responses from the three groups.

Results: 285 forms were returned-F 36\%, H 53\%, M 40\%. Advice to pregnant women on any nutritional supplement was given by F $89 \%, \mathrm{H} 76 \%, \mathrm{M} 85 \%$. Supplements advised were folic acid (F $90 \%, \mathrm{H} 82 \%, \mathrm{M} 89 \%$ ); iron (F 42\%, H 59\%, M 60\%); calcium (F $17 \%$, H $22 \%, M 18 \%$ ); vitamin D (F 12\%, H 17\%, M 10\%). In the past 3 years cases of suspected/proven vitamin $\mathrm{D}$ deficiency had been seen in pregnant Asian women by F 12\%, H 29\%, M 15\% and in other Asian adults by F $27 \%, \mathrm{H} 12 \%$. When asked whether they were convinced that pregnant Asian women should be offered calcium/ vitamin D supplements F 21\%, H 30\%, M 18\% agreed but a large majority were not sure (F 72\%, H 55\%, M42\%). When asked whether Asian women should be given routine vitamin D supplementation in pregnancy F 43\%, H 47\%, M 37\% agreed.
Conclusions: Most health carers are aware of the continuing problem of vitamin D deficiency in Asian pregnancies but there is confusion about recommendations and no clear prescription policy. Since the audit was completed specialist groups have met and in accordance with COMA recommendations have agreed on a policy of supplementing all pregnant Asian women with vitamin D 400 IU per day. An implementation programme is under way to help eradicate this eminently preventable condition.

\section{G105 VENTRICULO-PERITONEAL (VP) SHUNT MALFUNCTION: AN ANALYSIS OF PRESENTING SYMPTOMS AND SIGNS AND THEIR CORRELATION WITH SURGICAL FINDINGS}

N.P. Barnes, S.J. Jones, R.D. Hayward, W.J. Harkness, D. Thompson. Department of Neurosurgery, Great Ormond Street Hospital, London, UK

Aims: To evaluate the predictive value of the symptoms, signs and radiographic findings accompanying presumed shunt malfunction, by comparing presentation with operative findings.

Methods: A prospective study of all 53 patient referrals to a regional paediatric neurosurgical centre between April-November 1999 with a diagnosis of presumed shunt malfunction. Presenting symptoms and signs were recorded along with the results of CT scanning, operative findings and clinical outcome. Two patient groups were defined, one with proven shunt malfunction, the other with normal shunt function. Symptomatology and CT scan findings in each were then compared.

Results: Common presenting features were headache, drowsiness, vomiting and fever. CT scans were performed in all. 37 had operatively-proven shunt malfunction, of whom $84 \%$ had increased ventricle size when compared with previous imaging. For the 2 patient groups, (with, and without shunt malfunction) odds ratios with confidence intervals on their presenting symptoms were headache 1.5 $(0.27,10.9)$ vomiting $0.9(0.25,3.65)$ drowsiness $10(0.69,10.7)$ fever $0.19(0.03,6.95)$

Conclusions: This study re-iterates that drowsiness is by far the best clinical predictor of VP shunt malfunction. Headache and vomiting are poorly predictive of acute shunt block, while fever suggests an alternative diagnosis. CT findings should only be reliably interpreted when compared with previous CT examinations, as not all with proven acute shunt block will present with increased ventricle size.

\section{G106 WHAT PROPORTION OF ACUTE CHILD ATTENDERS TO ACCIDENT AND EMERGENCY COULD BE SEEN IN A COMMUNITY ASSESMENT UNIT?}

K. Armon ${ }^{1}$, T.J. Stephenson ${ }^{1}$, R. MacFaul ${ }^{2}$, P. Eccleston ${ }^{1}$, U. Werneke ${ }^{3}$. ${ }^{1}$ Child Health, University of Nottingham; ${ }^{2}$ Pinderfields General Hospital; ${ }^{3}$ Maudsley Hospital, London, UK

Introduction: Attendance of children to accident and emergency $(\mathrm{A} \& \mathrm{E})$ departments is rising. A community paediatric assessment unit may reduce these numbers and manage children more effectively.

Aim: To determine what proportion of children attending a paediatric (A\&E) department could be effectively seen in a community assessment unit.

Methods: Data were collected prospectively on all children (0-15 years) attending the only paediatric A\&E department in Nottingham (data included all emergency GP referrals) between Feb 1997 and Feb 1998. Patient group, admissions to hospital, investigations undertaken and triage scores were included.

Results: 38,982 children were seen, of which $26,756(71 \%)$ were classified as trauma or surgical, and were excluded as being appropriate to see in A\&E. 10,369 (28\%) were medical, 541 (1.4\%) other or did not wait and $1316(3.4 \%)$ could not be classified. Of the medical cases, $25 \%$ had been referred by a GP. 3250 (32\%) were admitted and therefore excluded. Of those not admitted, 23\% required an X-ray and an additional $11 \%$ a blood test, leaving 66\% (approx. 4698) requiring neither. Data for triage scores was incomplete, however approximately $8 \%$ were in triage categories 1 or 2 and therefore had to be seen by a doctor within 10 minutes. Thus approximately 4322 children could have been appropriately seen in a community assessment unit, $11 \%$ of the total children attending.

Conclusion: Of 39,000 children attending a paediatric $\mathrm{A} \& \mathrm{E}$ department over one year only $4322(11 \%)$ were appropriate to see within a community assessment centre with no laboratory or imaging facilities or inpatient beds. 


\section{G107 A POPULATION BASED STUDY OF DEHYDRATION WITH HYPERNATRAEMIA IN BREAST FED INFANTS}

S.J. Oddie, S. Richmond. Sunderland Royal Hospital, UK

Dehydration with hypernatraemia has been described as complicating exclusive breast feeding in at least 47 cases in the literature. As part of a geographically defined review of readmission to hospital in the neonatal period, we looked at cases of dehydration with hypernatraemia. Cases were exclusively breast fed, had a history of poor feeding and had weight loss corrected by rehydration. The serum sodium in cases was $150 \mathrm{mmol} / 1$ or above.

There were 928 readmissions to hospital during 1998. Of these 34 had lost more than $10 \%$ of their birthweight. Of these, eight met the case definition. All had lost more than $15 \%$ of birthweight. No baby had a subsequent diagnosis made which could have retrospectively explained the hypernatraemia. One infant had convulsions.

There were 32015 births in the region during 1998, giving a minimum population incidence of 2.5 per 10000 livebirths. This figure will underestimate the true incidence as this condition is almost confined to primiparous breastfeeding mothers. Breastfeeding rates are low in the Northern Region.

This study suggests that this disorder is not rare and represents an extreme form of unsuccessful breastfeeding. As with the other cases in the literature, the cardinal clinical feature of this problem is weight loss. Efforts to prevent this condition will benefit from strategies aimed at the promotion of successful breastfeeding if excessive weight loss is not ignored.

\section{G108 PSYCHIATRIC ASPECTS OF CHRONIC FATIGUE SYNDROME (CFS) IN CHILDHOOD: COMPARISON WITH JUVENILE ARTHRITIS (JIA) AND EMOTIONAL DISORDERS (ED)}

E. Garralda, L. Rangel, M. Levin. Academic Unit of Child Psychiatry, Imperial College School of Medicine, UK

Aims: to explore clinical specificity in CFS.

Methods: detailed standardised research interviews and Questionnaires with children and parents attending paediatric or psychiatric clinics with CFS $(\mathrm{N}=28)$, JIA $(\mathrm{N}=30)$, and ED (n-27). Mean age: 15 years; range: 11 to 18 years.

Results: Marked illness related impairment-including school non-attendance-was highest in CFS ( $87 \%$, vs 38\% JIA and 27 ED); psychopathology was higher in CFS than in JIA (one-year prevalence of $78 \%$ vs $26 \%$ ); personality problems were present in $66 \% \mathrm{ED}, 44 \%$ CFS and $12 \%$ JIA). Parental health problems were higher in CFS and ED than in JIA (maternal mental distress in $45 \%, 36 \%$ and $15 \%$ respectively), so was maternal emotional over-involvement with the child and family burden derived from the child's illness. Children with CFS had a pre-morbid history of repetitive infections $(21 \%$ vs $4 \%$ JIA and nil ED) and school absence: (29\% CFS vs $11 \%$ JIA and $9 \%$ ED).

Conclusions: functional illness-related impairment in psychologically vulnerable children would appear clinically specific to childhood CFS.

\section{Child Protection}

\section{G109 NEURORADIOLOGICAL ASPECTS OF SUBDURAL HAEMORRHAGES}

S. Datta, N. Stoodley, S. Jayawant, A.M. Kemp. Dept of Neuroradiology and Child Health, University of Wales College of Medicine, UK

Background: Most subdural haemorrhages (SDH) in infancy arise from Shaken Baby Syndrome, a severe form of child abuse. SDH has an incidence of $21 / 100,000$ in children under 2 years, (37/100,000 under 6 months). ${ }^{1} \mathrm{~A}$ diagnosis of SDH is confirmed on cranial imaging, principally CT or MRI. Despite American guidelines (AAP section on Radiology, 2000) children are incompletely investigated. ${ }^{1}$

Aims and method: We reviewed the neuroradiological findings of 50 children who sustained an SDH or subdural effusion when they were under two years of age between 1992-2000. All cases were reviewed by a Paediatric Neuroradiologist who was blinded to the original report. We identified specific radiological features associated with SDH secondary to physical child abuse and propose a protocol for neuroimaging in suspected SDH in infancy.

Results: Neuroradiological review of images identified abnormalities not previously reported. There was wide variation in protocols used for neuroimaging. In a significant number of cases MRI altered initial CT diagnosis or added information to support the diagnosis of NAI. Interhemispheric bleeds and SDH in multiple sites or of different ages were almost exclusively seen in NAI.

Conclusions: We recommend guidelines for neuroimaging suspected SDH in infants. Scans should be reported by a neuroradiologist with experience in NAI. First line investigation should be CT scan, MRI should be re-imaging technique of choice. CT should be considered in all infants referred for child protection investigation especially if under 6 months old or with neurological signs or retinal haemorrhage or fractures.

1. Jayawant $S$, et al. Subdural haemorrhage in infants: population based study. BMF 1998;317;1558-61.

\section{G110 ANOGENITAL WARTS AND SEXUAL ABUSE}

K.E. Rollison, C.Lazaro. Lindisfarne Centre, RVI, Newcastle upon Tyne, UK

Aims: To establish incidence of sexual abuse in children with anogenital warts and to identify factors associated with abuse.

Methods: Retrospective review of case notes from 1995-2000 of children with anogenital warts appearing before age 12 years.

Results: These are available on 75 /88 cases. 43 were girls. 48 were under three years when the warts appeared. In 25 sexual abuse was considered likely because there were diagnostic genital findings of abuse or serious concerns established by social services investigation. $17 / 25$ were girls and $15 / 25$ were less than three years old

Factors associated with likely abuse are shown in the table.

Abstract G110

\begin{tabular}{llll}
\hline & & Total & Abuse \\
\hline $\begin{array}{llll}\text { Family history of anogenital } \\
\text { warts }\end{array}$ & $\begin{array}{l}\text { in mother of child <3 } \\
\text { yrs }\end{array}$ & 14 & 2 \\
& $\begin{array}{l}\text { in mother of child } \geqslant 3 \\
\text { yrs }\end{array}$ & 8 & 5 \\
& in father & 10 & 2 \\
in mother's partner & 7 & 7 \\
Hand warts in preceding year & in the child & 18 & 5 \\
Site of warts in girls & perianal & 22 & 6 \\
& perianal + genital & 9 & 4 \\
& genital & 8 & 7 \\
& buttock or groin & 4 & 0 \\
\hline
\end{tabular}

Results of wart typing were presented.

Conclusion: There is a high incidence of abuse in children referred with anogenital warts -1 in 3 in our study. The likelihood of abuse is increased if warts are present in the genital area in girls $(\mathrm{p}<$ 0.01 ) or if there is a known history of warts in the mother's partner. Abuse should be considered even if there is a history of skin warts. All children with anogenital warts should be referred for specialised assessment. 